\title{
Excess serum uric acid is associated with metabolic syndrome in obese adolescent patients
}

\author{
Christy Foster ${ }^{1}$ (i) $\cdot$ Loretta Smith $^{2} \cdot$ Ramin Alemzadeh $^{2}$
}

Received: 15 July 2019 / Accepted: 6 February 2020 / Published online: 16 May 2020

(C) The Author(s) 2020

\begin{abstract}
Purpose Obesity is a significant cause of morbidity in adolescents. Excess serum uric acid (SUA) has been associated with metabolic syndrome (MS) among adults. We evaluated the relationship among SUA and markers of insulin resistance (IR) and low-grade inflammation in obese adolescents with and without MS.

Methods The study was a retrospective chart review of obese patients seen in the LeBonheur Endocrine clinic seen in clinic between September 2016 and December 2017. MS was defined as according to the International Diabetes Federation. Body mass index standard deviation score (BMI SDS), systolic blood pressure (SBP), diastolic blood pressure (DBP), body composition, fasting lipids, glucose, high sensitivity c-reactive protein (hs-CRP), serum uric acid (SUA), HbAlc, alanine transferase (ALT), aspartate transferase (AST), insulin and homeostatic model assessment for insulin resistance (HOMA-IR) were extracted from the charts of the 100 obese adolescents ( $57 \%$ female).

Results Hyperuricemia (SUA $>357$ umol/L) was present in $41.8 \%$ of entire cohort without significant ethnic/racial and/or gender differences. Adolescents with HUA had higher FM, SBP, HbA1c, insulin and HOMA-IR $(p<0.05)$. While SUA was positively correlated with FM, SBP, HOMA-IR and HbAlc, and triglyceride:HDL-C ratio (TG:HDL-C) $(\mathrm{p}<0.05)$. MS was identified in $32.8 \%$ of cohort. MS showed significantly higher FM, SBP, DBP, SUA, ALT, insulin, HOMA-IR, and TG:HDL-c ratio than non-MS subgroup $(p<0.05)$. FM was positively correlated with SUA, HOMA-IR and hsCRP $(p<0.01)$.

Conclusions In our study, those with hyperuricemia (HUA) showed elevated markers of metabolic syndrome including BP, serum glucoses, IR and triglycerides. In our cohort, SUA appears to correlate with MS comorbidities.
\end{abstract}

Keywords Hyperuricemia $\cdot$ Inflammation $\cdot$ Adolescents $\cdot$ Metabolic syndrome $\cdot$ Obesity

\section{Introduction}

Obesity has been identified as significant public health concern over the past couple decades [1]. Metabolic syndrome (MS) is a cluster of symptoms including central obesity, insulin resistance, hypertension, and hypercholesterolemia [2]. According to the World Health Organization, the worldwide prevalence of obesity in children has increased by more than

Christy Foster

chfoster@peds.uab.edu

1 Division of Endocrinology, Department of Pediatrics, University of Alabama at Birmingham, 1601 4th Avenue South, Birmingham, AL, USA

2 Division of Endocrinology, Department of Pediatrics, University of Tennessee Health Science Center, Memphis, TN, USA three fold over the past four decades [3]. Metabolic syndrome in children is a risk factor for development of Type 2 diabetes and adult MS [4], therefore more studies are needed to understand the pathophysiology of MS leading to inflammation. In addition, serum uric acid (SUA) has been found to be an independent risk factor for MS [5]. However, SUA homeostasis and its relationship with MS are considered complex [6]. High levels of SUA will lead to sequelae including hypertension [7]-[8], hypertriglyceridemia, and hypercholesterolemia $[5,8]$.

Uric acid is the end product of purine metabolism and is secreted by the kidney. Elevated levels of SUA can result from decreased renal clearance. Hyperinsulinemia has been postulated to decrease uric acid clearance by the kidneys and to increase serum uric acid [9]. Nitric oxide (NO) is the major endothelium-derived relaxing factor associated with oxidative stress and insulin resistance. Uric acid impairs endothelial function and enhances nitric synthase deficiency, which 
reduces NO, a known mechanism for inducing insulin resistance [10]. Also, SUA has been found to be associated with cardiovascular disease in adults with or without impaired glucose tolerance [11].

Hyperuricemia has been shown to be associated with MS in adult patients. Adults with increasing BMI over time show increased SUA and high sensitivity C-reactive protein (hsCRP) [12]. Serum uric acid has also been shown to correlate with liver dysfunction and increased inflammatory markers [10, 13-17] and negatively correlate with fasting blood glucose, serum HbA1c and HDL-C [18]. Prior studies define hyperuricemia at serum uric acid level as greater than $6.0 \mathrm{mg} / \mathrm{dL}$. [7]

In obese children, SUA, hsCRP and other inflammatory markers are elevated [19], which has been associated with excess weight and low cardiovascular fitness [20]. Prior studies have looked at SUA level in adolescent patients with metabolic syndrome and have shown a correlation between SUA and markers of inflammation and endothelial dysfunction in pre-pubertal children [19, 21]. It has been shown that markers of inflammation and SUA are associated with biomarkers of insulin resistance and sequela of MS in adolescents [21]. African American adults have significantly higher levels of SUA [22], whereas studies evaluating this in African American adolescents are scarce. Indeed, the metabolic interaction between SUA and low grade inflammation among adolescents has not been fully explored [23].Therefore, we examined the differences in SUA and hsCRP and indices of insulin resistance among adolescents with and without metabolic syndrome.

\section{Subjects and methods}

\section{Subjects and design}

The study was designed as a retrospective chart review of patients seen in the LeBonheur Endocrine clinic from September 2016 to December 2018 for obesity, identified by a practitioner seeing the patients in the LeBonheur clinic who had a weekly obesity clinic. Because this was a retrospective chart review, a sample size calculation was not done. One hundred adolescents (age 13.9-18.9 years old) who met the criteria for obesity [body mass index (BMI) $>95$ th percentile for age] were identified during this period of time. Race was self-assigned: Caucasian $(n=28)$ and African-American $(n=$ 72). Children were excluded if they had a diagnosis of diabetes given that this could be a confounding variable, hepatic or renal disease (renal disease defined as GFR $<60 \mathrm{ml} / \mathrm{min} /$ $1.73 \mathrm{~m} 2)$ metabolic rickets, malabsorptive disorders (Crohn's disease, cystic fibrosis, and celiac disease) or cancer or were taking anticonvulsants, or systemic glucocorticoids. The UTHSC Institutional Review Board committee approved the retrospective review of patients' clinical charts; therefore, informed consent was not required.

As part of routine care, participants and/or their guardians completed an intake form detailing their medical history and medications. A well-trained clinician determined pubertal maturation (Tanner stage). Data were collected on patients including age, gender, self-declared race, and anthropometric measurements such as BMI, height, weight, blood pressure, and body composition analysis. Waist circumferences (WC) were measured to the nearest $0.1 \mathrm{~cm}$ at the level of iliac crest while the patient was at minimal respiration. Body composition analysis was done using a Tanita DC $430 \mathrm{U}$ body composition impedance scale (Arlington Heights, IL) to measure fat mass (FM), fat free mass (FFM) and total body water (TBW) [24, 25]. Fasting samples for glucose, SUA, insulin, hemoglobin A1C, lipid profile, ALT, AST and hsCRP were obtained between 7 AM-11:30 AM. depending on the patient appointment time.

\section{Laboratory studies and calculations}

All blood samples were obtained between 7 AM and 11:30 AM after an overnight fast. Serum glucose. Was measured using an autoanalyzer (Ortho-Clinical Diagnostic, Inc. Rochester, NY). Fasting serum insulin was measured using the Architect insulin assay which is a chemiluminescent micro particle immunoassay. Intra-assay and inter-assay CV are 1.7$4.0 \%$ and $1.9-4.6 \%$ respectively. The homeostatic model assessment of insulin resistance (HOMA-IR) [(blood glucose, $\mathrm{mM} \mathrm{X}$ insulin, $\mathrm{uU} / \mathrm{mL}) / 22.5$ ] was calculated [26]. Hemoglobin A1c was determined using a Vitros Chemistry instrument (non-diabetic range 4\%-6\%).

Total cholesterol, high-density lipoprotein cholesterol (HDL-C), low-density lipoprotein cholesterol (LDL-C) and triglycerides were determined by colorimetric methods (Vitros 5600 Integrated System; Ortho-Clinical Diagnostics, Inc. Rochester, NY). Low density lipoprotein cholesterol was calculated using Friedewald's equation [27]. AST and ALT were determined by colorimetric methods (Vitros 5600 Integrated System; Ortho-Clinical Diagnostics, Inc. Rochester, NY).

SUA levels were measured by oxidation with enzyme uricase to form allantoin and hydrogen peroxide by a colorimetric assay (Vitros 5600 Integrated system; Ortho-Clinical Diagnostics, Inc. Rochester, NY). Hyperuricemia was defined as levels above the measurement of $357 \mathrm{uM}(6.0 \mathrm{mg} / \mathrm{dL})$ for adolescents aged 13-18 years old as shown in a prior study [7].

Serum hsCRP level was determined by an immunoassay using a polystyrene particle-enhanced immunonephelometric method (Vitros 5600 Integrated system; Ortho-Clinical Diagnostics, Inc. Rochester, NY) The limit of detection was $0.1 \mathrm{mg} / \mathrm{L}$ with a measuring range of $0.2-15.0 \mathrm{mg} / \mathrm{L}$. Inter- 
assay CV was $1.2-5.0 \%$. The hs-CRP values of $>10 \mathrm{mg} / \mathrm{L}$ were excluded to avoid influence of acute infection [28].

Blood pressure measurements were taken twice with the patient in the sitting position. Elevated systolic blood pressure (SBP) or diastolic blood pressure (DBP) were defined as a value above the 95 th percentile for age, gender and height percentile.

MS was defined according to a consensus statement from the International Diabetes Federation. The IDF defines MS as central obesity (defined as a waist circumference $>95$ th percentile) and two of the following: Triglycerides $>150 \mathrm{mg} / \mathrm{dL}$, HDL-C $<40 \mathrm{mg} / \mathrm{dL}$ in males or $<50 \mathrm{mg} / \mathrm{dL}$ in females, elevated blood pressure (BP $>95$ th percentile based on height, age and gender) and fasting plasma glucose $>100 \mathrm{mg} / \mathrm{dL}$ [29]]. Adjustments were made regarding the definition of hypertension given that these were children and adolescents in the study. Impaired fasting glucose was defined as glucose between $5.6 \mathrm{mmol} / \mathrm{L}$ to $6.9 \mathrm{mmol} / \mathrm{L}$.

\section{Statistical analyses}

Statistical analyses were carried out using SPSS 25. Data are expressed as mean \pm standard deviation. BMI values were converted into standard deviations scores (SDS) that were normalized for age and gender based on 2000 Centers for Disease Control (CDC) growth charts. The natural logarithmic transformation of the variables was used in the correlation and regression analyses when the data was found to be skewed. Differences between normal uric acid (NUA) and high uric acid (HUA) groups and those with MS and non-MS groups were estimated using unpaired student t-tests and the $p$ value for 2-tailed significance given the direction of the change between the groups was unknown. Chi-squared analyses were used to compare prevalence of hypertension and metabolic syndrome. Spearman correlations were performed to examine associations between SUA, hsCRP, FM, blood pressure, HOMA-IR and triglycerides:cholesterol ratio and triglycerides:HDL ratio. $P<0.05$ was considered significant.

\section{Results}

\section{Findings stratified according to presence or absence of elevated serum uric acid}

Hyperuricemia was identified in $39 \%$ of the cohort. Those patients with HUA also were found to have significantly higher SBP and DBP, BMI SDS, WC, and FM. Total Body Water (TBW) and Free Fat Mass (FFM). Patients with HUA had significantly higher fasting glucose, fasting insulin, HOMA-IR, GFR, and total cholesterol and LDL-C levels compared to those with NUA. Serum aspartate aminotransferase (AST) levels were also significantly higher in those with high SUA (Table 1).

Looking at those who were in the lowest and highest quartile of SUA, there was significant difference between BMI SDS, FM, FFM, SBP, and cholesterol/HDL ratio. In the highest quartile of SUA, hsCRP correlated positively with serum glucose. SUA did not correlate with FM, HOMA-IR or SBP (data not shown).

\section{Findings stratified according to presence or absence of metabolic syndrome}

In our cohort, $30 \%$ of adolescents met diagnostic criteria for MS. Patients with MS were shown to have significantly higher SBP, DBP, BMI SDS, WC, FM, fasting insulin level, SUA, ALT, total cholesterol, LDL-C, and HOMA-IR. (Table 2).There was no significant difference in GFR between those with metabolic syndrome and those without metabolic syndrome.

\section{Findings stratified according to ethnicity/race and gender}

There were significant differences in BMI SD, fasting glucoses, triglycerides, and hemoglobin Alc among ethnic groups. While African American subjects had higher BMI SDS and hemoglobin A1C values compared to Caucasians, fasting serum glucose levels were found to be significantly higher among Caucasians compared to African-American patients (Table 3).

Fourty $-3 \%$ of the cohort were male and $57 \%$ were female (Table 4). Female subjects had higher pubertal maturation staging of the males and females. Female subjects were found to have significantly higher fat percentage, lower FFM/FM ratio (data not shown) whereas males were found to have higher average fasting serum glucose levels and AST/ALT ratio (data not shown).

\section{Findings in the entire cohort}

Table 5 provides a summary of bivariate correlations among the clinical and biochemical variables for the entire cohort. FM was found to correlate with SBP and DBP as well as HOMA-IR, triglycerides:HDL-C ratio, and hsCRP. SUA were shown to correlate with FM, HOMA-IR, triglycerides:HDL-C levels, systolic and diastolic blood pressure (Fig. 1). hsCRP was not significantly positively correlated with SUA in the entire cohort. Interestingly, hs-CRP was not correlated with the triglcyerides:HDL or HOMA-IR. All patients had a GFR $>60 \mathrm{ml} / \mathrm{min} / 1.73 \mathrm{~m}^{2}$. Mean GFR was $107 \mathrm{ml} / \mathrm{min} / 1.73 \mathrm{~m}^{2}$.

Six subjects were identified with impaired fasting glucose. These patients had significant elevation in HOMA-IR, fasting 
Table 1 Clinical and biochemical characteristics of participants based on uric acid status

\begin{tabular}{lllll}
\hline Parameters & All & HUA & NUA & $P$ \\
& & $(\geq 357$ umol/L) & $(<357$ umol/L) & \\
\hline N (\%) & 100 & 39 & 61 & NA \\
Age (yrs) & $13.9 \pm 2.0$ & $14.4 \pm 2.1$ & $13.6 \pm 2.0$ & 0.049 \\
Gender (\% Female) & $57 \%$ & $46 \%$ & $64 \%$ & NS \\
Tanner Stage & $4.0 \pm 1.3$ & $4.4 \pm 1.0$ & $3.8 \pm 1.4$ & 0.031 \\
Race/Ethnicity & & & & \\
Caucasians (\%) & $28 \%$ & $23 \%$ & $31 \%$ & NS \\
African Americans(\%) & $72 \%$ & $77 \%$ & $69 \%$ & NS \\
SBP (mmHg) & $116.8 \pm 10.8$ & $122.2 \pm 11.3$ & $113.4 \pm 9.0$ & $<0.001$ \\
DBP (mmHg) & $71.9 \pm 8.1$ & $73.9 \pm 9.9$ & $70.6 \pm 6.4$ & $<0.001$ \\
Hypertension \% & $17 \%$ & $28 \%$ & $10 \%$ & 0.017 \\
BMI SDS & $2.4 \pm 0.4$ & $2.5 \pm 0.3$ & $2.3 \pm 0.4$ & $<0.001$ \\
Waist Circumference (cm) & $108.1 \pm 15.4$ & $113.4 \pm 13.5$ & $104.7 \pm 15.6$ & 0.004 \\
Fat Mass (FM) & $42.8 \pm 17.6$ & $48.8 \pm 17.7$ & $39.0 \pm 16.4$ & 0.008 \\
Glucose (mmol/L) & $4.8 \pm 0.5$ & $4.9 \pm 0.5$ & $4.7 \pm 0.4$ & 0.038 \\
Insulin (pmol/L) & $194.4 \pm 157.2$ & $244.3 \pm 156.0$ & $162.5 \pm 150.8$ & 0.011 \\
HOMA-IR (mol uU/mL) & $5.81 \pm 4.85$ & $7.50 \pm 5.19$ & $4.72 \pm 4.32$ & 0.007 \\
GFR (ml/min/1.73 m2) & $107.7 \pm 19.37$ & $101.7 \pm 18.50$ & $111.60 \pm 19.08$ & 0.013 \\
AST & $29.7 \pm 10.5$ & $33.1 \pm 12.6$ & $27.5 \pm 8.32$ & 0.017 \\
ALT & $35.8 \pm 16.1$ & $38.05 \pm 16.0$ & $34.4 \pm 16.2$ & NS \\
hs-CRP (mg/L) & $2.8 \pm 2.2$ & $3.0 \pm 2.3$ & $2.6 \pm 2.1$ & NS \\
SUA (umol/L) & $341.5 \pm 76.3$ & $414.1 \pm 51.8$ & $295.2 \pm 47.9$ & $<0.001$ \\
Cholesterol (mmol/L) & $4.0 \pm 0.8$ & $4.2 \pm 1.0$ & $3.9 \pm 0.7$ & NS \\
Triglycerides (mmol/L) & $1.2 \pm 0.6$ & $1.3 \pm 0.6$ & $1.1 \pm 0.6$ & NS \\
Triglycerides: HDL-C Ratio & $2.5 \pm 1.5$ & $2.6 \pm 1.3$ & $2.4 \pm 1.6$ & NS \\
Prevalence of MS (\%) & $30 \%$ & $46 \%$ & $20 \%$ & 0.005 \\
\hline & & & & \\
\hline
\end{tabular}

Abbreviations: Not applicable (NA), not significant (NS), serum uric acid (SUA), normal uric acid (NUA), high uric acid (HUA) serum glucose and serum A1c compared to those without impaired glucose tolerance. Interestingly, looking at these patients, uric acid positively correlated with insulin levels, FM, HOMA-IR, SBP and DBP. High-sensitivity CRP correlated with FM but was not positively correlated to SUA (Fig. 1).

\section{Discussion}

In the present study, elevated SUA was identified in $46 \%$ of the entire cohort and was positively correlated with higher triglcyerides;HDL-C and HOMA-IR, an index of insulin resistance. Females were found to have significantly higher FM and serum insulin levels than males. Patients with MS were shown to have significantly higher SUA as well as significantly higher SBP and DBP, fasting serum insulin and cholesterol. Patients with hyperuricemia had a higher prevalence of MS. These findings are consistent with prior studies showing that hyperuricemia is an independent risk factor associated with the development of MS $[18,30]$.
Hyperuricemia can impair vascular function by exerting pro-oxidant effects and by decreasing $\mathrm{NO}$ bioavailability which likely induces hypertension and MS [31]. In adults, SUA has been seen as a biomarker for cardiometabolic risk [32]. Prior studies in adults have shown that participants with elevated SUA had a higher incidence of diabetes and pre diabetes in adults [33]. Studies has shown that hyperuricemia alone, independent of obesity, can increase the risk of hypertension [34]. Indeed, SUA was found to be associated with elevated blood pressure in our cohort. Studies which have looked at the incidence of gout determined that obesity was independently associated with gout and hyperuricemia in women $[35,36]$ Our current study also reinforces that those with MS had increased SUA levels.

In our study, the incidence of hyperuricemia was observed in $39 \%$ in our population. The prevalence of hyperuricemia among adolescents with MS is variable among different populations [37]. Ford et al. reported hyperuricemia prevalence of $59.9 \%$ among US children and adolescents using SUA level of $334 \mathrm{uM}$. Tang et al. observed a prevalence of $30.5 \%$ in 
Table 2 Clinical and Biochemical Characteristics of Participants with and without MS

\begin{tabular}{|c|c|c|c|c|}
\hline Parameters & All & MS & Non-MS & $\boldsymbol{P}$ \\
\hline $\mathrm{N}$ & 100 & 30 & 70 & \\
\hline Age (yrs) & $13.9 \pm 2.0$ & $14.0 \pm 2.1$ & $13.9 \pm 2.0$ & NS \\
\hline Gender (\% Female) & $57 \%$ & $67 \%$ & $53 \%$ & NS \\
\hline Tanner Stage & $4.0 \pm 1.3$ & $4.2 \pm 1.1$ & $3.9 \pm 1.3$ & NS \\
\hline \multicolumn{5}{|l|}{ Race/Ethnicity } \\
\hline Caucasians (\%) & $28 \%$ & $36 \%$ & $23 \%$ & NS \\
\hline African Americans (\%) & $72 \%$ & $63.3 \%$ & $75.7 \%$ & NS \\
\hline $\mathrm{SBP}(\mathrm{mmHg})$ & $116.81 \pm 10.84$ & $123.6 \pm 13.79$ & $113.9 \pm 7.75$ & 0.001 \\
\hline DBP (mmHg) & $71.9 \pm 8.1$ & $75.6 \pm 9.8$ & $70.3 \pm 6.7$ & 0.011 \\
\hline$\%$ Hypertension & $17 \%$ & $37 \%$ & $9 \%$ & 0.001 \\
\hline BMI SDS & $2.4 \pm 0.4$ & $2.5 \pm 0.3$ & $2.3 \pm 0.4$ & 0.013 \\
\hline Waist circumference & $108.1 \pm 15.4$ & $112.7 \pm 12.8$ & $106.2 \pm 16.0$ & 0.033 \\
\hline Fat Mass (kg) & $42.8 \pm 17.6$ & $48.48 \pm 16.2$ & $40.3 \pm 17.6$ & 0.028 \\
\hline Glucose & $4.8 \pm 0.5$ & $4.9 \pm 0.6$ & $4.7 \pm 0.4$ & NS \\
\hline Insulin (pmol/L) & $194.4 \pm 157.2$ & $296.1 \pm 218.4$ & $150.8 \pm 94.0$ & 0.001 \\
\hline HOMA-IR & $5.8 \pm 4.9$ & $8.7 \pm 6.7$ & $4.5 \pm 2.9$ & 0.001 \\
\hline AST & $29.7 \pm 10.5$ & $31.9 \pm 10.7$ & $28.8 \pm 10.4$ & NS \\
\hline ALT & $35.8 \pm 16.1$ & $43.8 \pm 22.2$ & $32.4 \pm 11.3$ & 0.011 \\
\hline hs-CRP (mg/L) & $2.7 \pm 2.2$ & $3.1 \pm 2.1$ & $2.6 \pm 2.2$ & NS \\
\hline SUA (umol/L) & $341.5 \pm 76.3$ & $370.8 \pm 77.4$ & $329.0 \pm 72.8$ & 0.009 \\
\hline Cholesterol (mmol/L) & $4.0 \pm 0.8$ & $4.5 \pm 0.9$ & $3.8 \pm 0.8$ & $<0.001$ \\
\hline Triglycerides (mmol/L) & $1.2 \pm 0.6$ & $1.64 \pm 0.7$ & $0.98 \pm 0.4$ & $<0.001$ \\
\hline Triglycerides: HDL-C Ratio & $2.5 \pm 1.5$ & $3.7 \pm 0.9$ & $1.9 \pm 0.9$ & $<0.001$ \\
\hline
\end{tabular}

Abbreviations: fat mass (FM), homeostatic model assessment estimates for insulin resistance (HOMA-IR), metabolic syndrome (MS), not applicable (NA) and not significant (NS)
Table 3 Clinical and biochemical Characteristics of participants according to ethnicity/race

\begin{tabular}{llll}
\hline Ethnicity & Caucasians & African American & $\mathrm{P}$ \\
\hline $\mathrm{N}$ & 28 & 72 & \\
Age & $13.9 \pm 2.0$ & $13.9 \pm 2.1$ & $\mathrm{NS}$ \\
Gender (\% Female) & $57 \%$ & $57 \%$ & $\mathrm{NS}$ \\
Hypertension \% & $19 \%$ & $17 \%$ & $\mathrm{NS}$ \\
BMI SDS & $2.2 \pm 0.4$ & $2.4 \pm 0.3$ & 0.034 \\
Waist Circumference (cm) & $104.3 \pm 17.6$ & $109.6 \pm 14.3$ & $\mathrm{NS}$ \\
Fat Mass (kg) & $37.8 \pm 14.3$ & $44.7 \pm 18.3$ & $\mathrm{NS}$ \\
Glucose (mmol/L) & $4.9 \pm 0.5$ & $4.7 \pm 0.5$ & 0.01 \\
Insulin (pmol/L) & $205.6 \pm 162.0$ & $193.0 \pm 157.9$ & $\mathrm{NS}$ \\
HOMA-IR & $6.5 \pm 5.7$ & $5.6 \pm 4.6$ & $\mathrm{NS}$ \\
hs-CRP (mg/L) & $2.75 \pm 2.09$ & $2.76 \pm 2.19$ & $\mathrm{NS}$ \\
SUA (mg/dL) & $336.9 \pm 59.7$ & $339.7 \pm 87.0$ & $\mathrm{NS}$ \\
Cholesterol (mmol/L) & $4.0 \pm 1.1$ & $4.0 \pm 0.7$ & $\mathrm{NS}$ \\
Triglycerides (mmol/L) & $1.43 \pm 0.71$ & $1.08 \pm 0.49$ & 0.006 \\
Triglycerides:HDL-C ratio & $2.95 \pm 1.9$ & $2.29 \pm 1.3$ & $\mathrm{NS}$ \\
Prevalence of MS (\%) & $39 \%$ & $26 \%$ & $\mathrm{NS}$ \\
\hline
\end{tabular}

Abbreviations: fat mass (FM), homeostatic model assessment estimates for insulin resistance (HOMA-IR), metabolic syndrome (MS), not applicable (NA) and not significant (NS)
Japanese children using a SUA cutoff level of $416 \mathrm{uM}$ $(7.0 \mathrm{mg} / \mathrm{dL})$ for male and $369(6.2 \mathrm{mg} / \mathrm{dL}) \mathrm{uM}$ in males and females. These studies show incidences which are different from in our study, most likely due to different SUA cutoffs. In our study, those with MS, SUA levels were significantly higher compared to those patients who did not meet criteria for MS. These findings are consistent with a prior study that looked at adolescents and hyperuricemia [38]. Similar to other studies, no gender differences in the prevalence of hyperuricemia in study participants were observed. Limited data in adolescents is available for evaluation of the relationship between hyperuricemia and the connection with inflammation in MS.

Of note, there were differences in the ethnicities between glucoses and hemoglobin A1C in our study with white participants having higher fasting glucose levels with AfricanAmerican participants having a higher hemoglobin A1C values. Based on other studies, African-American persons have higher hemoglobin A1C levels across the full spectrum of glucose levels secondary to differences in rates of glucose transport and glycosylation of the erythrocyte membrane [39, 40]. This difference could explain the findings in our study regarding average hemoglobin $\mathrm{A} 1 \mathrm{C}$. 
Table 4 Clinical and Biochemical Characteristics of Participants Based on Gender Abbreviations: fat mass (FM), homeostatic model assessment estimates for insulin resistance (HOMA-IR), metabolic syndrome (MS), not applicable (NA) and not significant (NS)

\begin{tabular}{lllll}
\hline Parameters & All & Male & Female & $P$ \\
\hline $\mathrm{N}$ & 100 & 43 & 57 & NA \\
Age (yrs) & $13.9 \pm 2.0$ & $13.6 \pm 1.9$ & $14.2 \pm 2.1$ & NS \\
Tanner Stage & $4.0 \pm 1.3$ & $3.4 \pm 1.6$ & $4.3 \pm 0.9$ & 0.005 \\
Race/Ethnicity & & & & \\
Caucasians (\%) & $28 \%$ & $28 \%$ & $28 \%$ & NS \\
African Americans (\%) & $72 \%$ & $72 \%$ & $72 \%$ & NS \\
Hypertension \% & $17 \%$ & $14 \%$ & $19 \%$ & NS \\
BMI SDS & $2.4 \pm 0.4$ & $2.4 \pm 0.3$ & $2.3 \pm 0.4$ & NS \\
Waist circumference & $104.5 \pm 18.0$ & $108.6 \pm 14.5$ & $107.8 \pm 16.1$ & NS \\
Fat Mass (kg) & $42.8 \pm 17.6$ & $38.1 \pm 14.3$ & $46.3 \pm 18.9$ & 0.015 \\
Glucose (mmol/L) & $4.8 \pm 0.5$ & $5.0 \pm 0.4$ & $4.6 \pm 0.5$ & 0.001 \\
HbA $(\%)$ & $5.5 \pm 0.3$ & $5.5 \pm 0.3$ & $5.5 \pm 0.2$ & NS \\
Insulin (pmol/L) & $194.4 \pm 157.2$ & $187.7 \pm 145.6$ & $199.5 \pm 166.6$ & NS \\
HOMA-IR (mol uU/mL) & $5.8 \pm 4.9$ & $5.9 \pm 4.9$ & $5.8 \pm 4.9$ & NS \\
AST & $29.7 \pm 10.5$ & $31.2 \pm 9.8$ & $28.6 \pm 11.0$ & NS \\
ALT & $35.8 \pm 16.1$ & $38.7 \pm 16.0$ & $33.6 \pm 16.0$ & NS \\
hs-CRP (mg/L) & $2.8 \pm 2.2$ & $2.8 \pm 2.3$ & $2.8 \pm 2.0$ & NS \\
SUA (umol/L) & $341.5 \pm 76.3$ & $357.7 \pm 75.8$ & $329.3 \pm 75.0$ & NS \\
Cholesterol (mmol/L) & $4.0 \pm 0.8$ & $3.9 \pm 0.7$ & $4.2 \pm 0.9$ & NS \\
Triglycerides (mmol/L) & $1.2 \pm 0.6$ & $1.1 \pm 0.53$ & $1.2 \pm 0.62$ & NS \\
Triglycerides:HDL-C ratio & $2.5 \pm 1.5$ & $2.5 \pm 1.4$ & $2.5 \pm 1.6$ & NS \\
Prevalence of MS (\%) & $30 \%$ & $23 \%$ & $35 \%$ & NS \\
\hline & & & & \\
\hline
\end{tabular}

Prior studies have shown that there is an increased incidence of elevated hsCRP in those who are obese. Our study did not show direct correlation between SUA and hsCRP, as other studies had shown. Our postulation is that perhaps the assay for hsCRP used during our study was different from the assay used in other studies. Another potential explanation is that with our study, since we were only evaluating obese patients, there was not enough variation in the SUA to detect a correlation of hsCRP because the values were all at one end of the spectrum.

Limitations to this study include retrospective design of the study and lack of glucose tolerance data to assess glucose homeostasis and beta-cell function and its relationship with SUA. In our study, there were also no age and sex-matched normal weight controls for each racial group to assess the correlation of hsCRP to SUA. These controls may help assess correlations between inflammatory markers and hyperuricemia. In addition, the accuracy of bioelectrical impedance analysis (BIA) for assessment of body composition has been questioned because of larger errors in individual estimates of body composition in large groups of normal weight or obese pediatric subjects compared with DXA [41]. However, BIA has been deemed accurate and has been previously shown to correlate DXA to measure body fat percentage in normal and

Table 5 Bivariate correlations of clinical and biochemical characteristics for whole cohort of participants

\begin{tabular}{|c|c|c|c|c|c|c|c|}
\hline & $\begin{array}{l}\mathrm{FM} \\
(\mathrm{kg})\end{array}$ & $\begin{array}{l}\text { SBP } \\
(\mathrm{mmHg})\end{array}$ & $\begin{array}{l}\text { DBP } \\
(\mathrm{mmHg})\end{array}$ & $\begin{array}{l}\text { SUA } \\
\text { (umol/L) }\end{array}$ & TG: HDL-C & $\mathrm{HbA}_{1 \mathrm{c}}(\%)$ & $\begin{array}{l}\text { HOMA-IR } \\
(\mathrm{mol} \mathrm{uU} / \mathrm{mL})\end{array}$ \\
\hline \multicolumn{8}{|l|}{$\mathrm{FM}(\mathrm{kg})$} \\
\hline $\mathrm{SBP}(\mathrm{mmHg})$ & 0.192 & & & & & & \\
\hline $\mathrm{DBP}(\mathrm{mmHg})$ & $0.323 * * *$ & $0.616 * * *$ & & & & & \\
\hline SUA (umol/L) & $0.335 * * *$ & $0.414 * *$ & $0.263 * *$ & & & & \\
\hline TG: HDL-C & $0.208 *$ & $.225^{*}$ & 0.076 & $0.224 *$ & & & \\
\hline $\mathrm{HbA}_{1 \mathrm{c}}(\%)$ & 0.049 & 0.099 & 0.076 & 0.149 & -0.011 & & \\
\hline HOMA-IR (mol uU/mL) & $0.237 *$ & $0.319 * * *$ & 0.202 & $0.247 *$ & $0.228^{*}$ & 0.196 & \\
\hline hs-CRP (mg/mL) & $0.263 * *$ & 0.055 & 0.034 & 0.06 & 0.05 & -0.114 & -0.046 \\
\hline
\end{tabular}

$* \mathrm{p}<0.05 ; * * \mathrm{p}<0.01 ; * * * p<0.001$ 
Fig. 1 Spearman correlations between uric acid and markers of metabolic syndrome. A. uric acid vs. fat mass. B. uric acid vs. HOMA-IR C. uric acid vs. triglyceride:HDL ratio D. Highsensitivity CRP vs. Fat mass. E. uric acid vs. systolic blood pressure F. uric acid vs. diastolic blood pressure
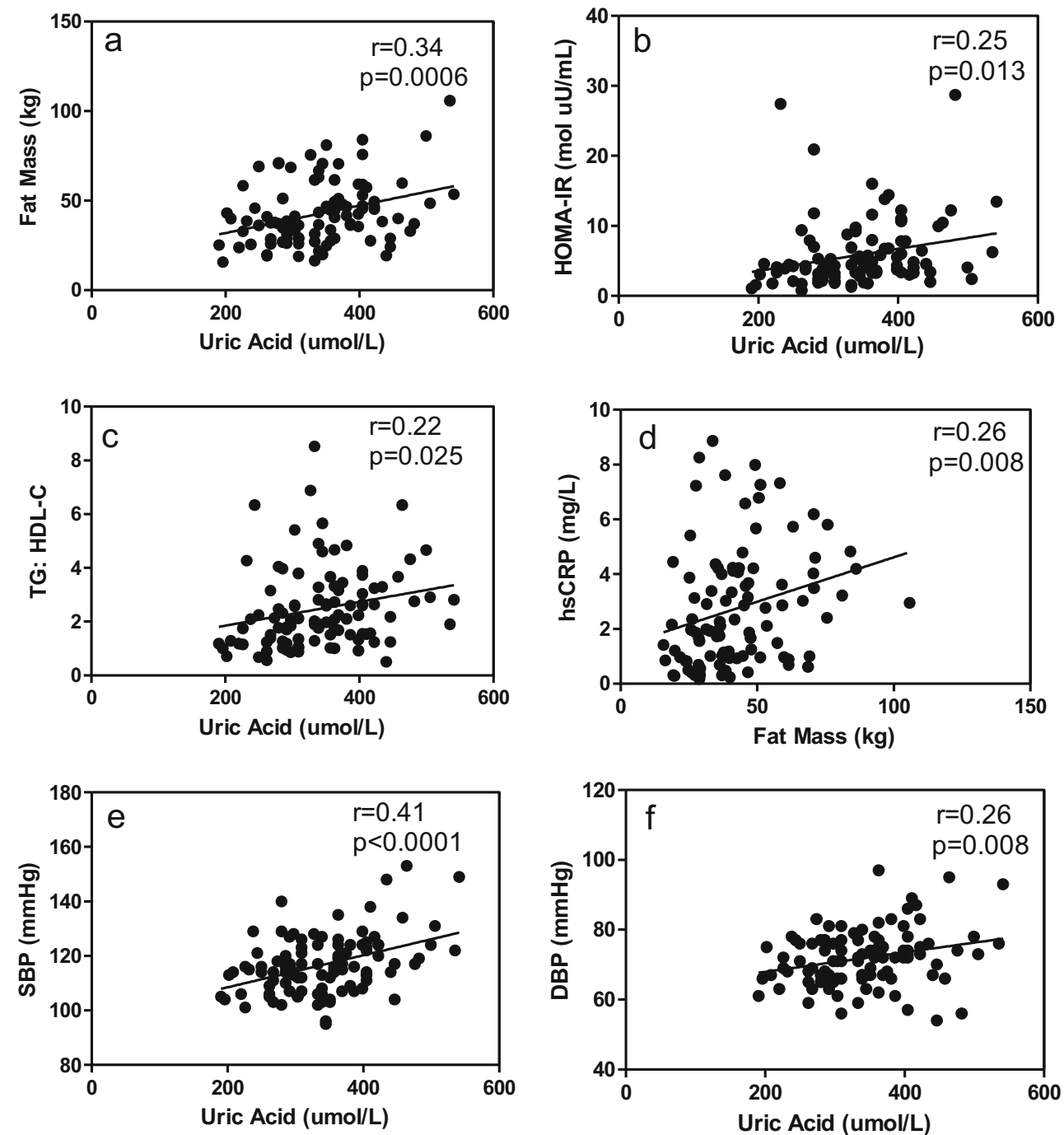

overweight patients. [42-44] However, recent studies have suggested BIA can under-estimate body fat in obese Asian individuals, which could be a limitation of this method. [45] Since we did not have any subject of Asian background, this limitation is not applicable. Therefore, we utilized the body impedance scale to assess body fat percentage. Exclusion of conditions which may affect uric acid was not done for this study, therefore it is a limitation and further study may be needed to look specifically into conditions such as asthma.

In conclusion, patients with hyperuricemia demonstrated significant elevations in markers of metabolic syndrome including higher blood pressures, serum glucoses, insulin resistance and triglycerides. Our study showed that patients with hyperuricemia have significantly higher fat mass on average. Our study confirmed the monotonic correlation of fat mass and uric acid in our population, which was composed predominately of African-American patients, which may not reflect a linear correlation. Additional studies are needed to further understand the relationship between hyperuricemia and markers of inflammation in the adolescent population with and without metabolic syndrome.

\section{Compliance with ethical standards}

Conflict of interest The authors have no conflict of interest to disclose.

Open Access This article is licensed under a Creative Commons Attribution 4.0 International License, which permits use, sharing, adaptation, distribution and reproduction in any medium or format, as long as you give appropriate credit to the original author(s) and the source, provide a link to the Creative Commons licence, and indicate if changes were made. The images or other third party material in this article are included in the article's Creative Commons licence, unless indicated otherwise in a credit line to the material. If material is not included in the article's Creative Commons licence and your intended use is not permitted by statutory regulation or exceeds the permitted use, you will need to obtain permission directly from the copyright holder. To view a copy of this licence, visit http://creativecommons.org/licenses/by/4.0/. 


\section{References}

1. Flegal KM, Kruszon-Moran D, Carroll MD, Fryar CD, Ogden CL. Trends in obesity among adults in the United States, 2005 to 2014. JAMA. 2016;315(21):2284-91.

2. Huang PL. A comprehensive definition for metabolic syndrome. Dis Model Mech. 2009;2(5-6):231-7.

3. Vikram NK. Cardiovascular and metabolic complications - diagnosis and Management in Obese Children. Indian J Pediatr. 2018;85(7):535-45.

4. Morrison JA, Friedman LA, Wang P, Glueck CJ. Metabolic syndrome in childhood predicts adult metabolic syndrome and type 2 diabetes mellitus 25 to 30 years later. J Pediatr. 2008;152(2):201-6.

5. Loeffler LF, Navas-Acien A, Brady TM, Miller ER 3rd, Fadrowski JJ. Uric acid level and elevated blood pressure in US adolescents: National Health and nutrition examination survey, 1999-2006. Hypertension. 2012;59(4):811-7.

6. Codoner-Franch $\mathrm{P}$, et al. Nitric oxide production is increased in severely obese children and related to markers of oxidative stress and inflammation. Atherosclerosis. 2011;215(2):475-80.

7. Alemzadeh R, Kichler J. Uric acid-induced inflammation is mediated by the parathyroid hormone:25-Hydroxyvitamin $\mathrm{D}$ ratio in obese adolescents. Metab Syndr Relat Disord. 2016;14(3):167-74.

8. Han GM, Gonzalez S, DeVries D. Combined effect of hyperuricemia and overweight/obesity on the prevalence of hypertension among US adults: result from the National Health and nutrition examination survey. J Hum Hypertens. 2014;28(10):579-86.

9. Baldwin W, McRae S, Marek G, Wymer D, Pannu V, Baylis C, et al. Hyperuricemia as a mediator of the proinflammatory endocrine imbalance in the adipose tissue in a murine model of the metabolic syndrome. Diabetes. 2011;60(4):1258-69.

10. Li C, Hsieh MC, Chang SJ. Metabolic syndrome, diabetes, and hyperuricemia. Curr Opin Rheumatol. 2013;25(2):210-6.

11. Wijnands JM, et al. The cross-sectional association between uric acid and atherosclerosis and the role of low-grade inflammation: the CODAM study. Rheumatology (Oxford). 2014;53(11):2053-62.

12. Hulsegge G, Herber-Gast GC, Spijkerman AM, Susan H, Picavet J, van der Schouw Y, et al. Obesity and age-related changes in markers of oxidative stress and inflammation across four generations. Obesity (Silver Spring). 2016;24(6):1389-96.

13. Kawamoto R, et al. An association between body mass index and high-sensitivity C-reactive protein concentrations is influenced by age in community-dwelling persons. Ann Clin Biochem. 2013;50(Pt 5):457-64.

14. Ferrara LA, Wang H, Umans JG, Franceschini N, Jolly S, Lee ET, et al. Serum uric acid does not predict incident metabolic syndrome in a population with high prevalence of obesity. Nutr Metab Cardiovasc Dis. 2014;24(12):1360-4.

15. Wu SH, Shu XO, Milne G, Xiang YB, Zhang X, Cai Q, et al. Uric acid correlates to oxidation and inflammation in opposite directions in women. Biomarkers. 2015;20(4):225-31.

16. Liu ST, et al. Obesity prevention practices and policies in child care settings enrolled and not enrolled in the child and adult care food program. Matern Child Health J. 2016;20(9):1933-9.

17. Leon-Pedroza JI, Escobedo G, Gonzalez-Chavez A. Relationship of hyperuricemia with low density lipoprotein, liver function tests and markers of systemic inflammation in patients with morbid obesity. Gac Med Mex. 2017;153(Supl. 2):S42-50.

18. Bonakdaran S, Kharaqani B. Association of serum uric acid and metabolic syndrome in type 2 diabetes. Curr Diabetes Rev. 2014;10(2):113-7.

19. Valle M, et al. Association of serum uric acid levels to inflammation biomarkers and endothelial dysfunction in obese prepubertal children. Pediatr Diabetes. 2015;16(6):441-7.
20. Reis LN, et al. Hyperuricemia is associated with low cardiorespiratory fitness levels and excess weight in schoolchildren. J Pediatr. 2017;93(5):538-43.

21. Wasilewska A, Tenderenda E, Taranta-Janusz K, Tobolczyk J, Stypułkowska J. Markers of systemic inflammation in children with hyperuricemia. Acta Paediatr. 2012;101(5):497-500.

22. Zhu Y, Pandya BJ, Choi HK. Prevalence of gout and hyperuricemia in the US general population: the National Health and nutrition examination survey 2007-2008. Arthritis Rheum. 2011;63(10): 3136-41.

23. Yu TY, Jee JH, Bae JC, Jin SM, Baek JH, Lee MK, et al. Serum uric acid: a strong and independent predictor of metabolic syndrome after adjusting for body composition. Metabolism. 2016;65(4):432-40.

24. Kettaneh A, et al. Reliability of bioimpedance analysis compared with other adiposity measurements in children: the FLVS II study. Diabetes Metab. 2005;31(6):534-41.

25. Karelis AD, et al. Validation of a portable bioelectrical impedance analyzer for the assessment of body composition. Appl Physiol Nutr Metab. 2013;38(1):27-32.

26. Matthews DR, Hosker JP, Rudenski AS, Naylor BA, Treacher DF, Turner RC. Homeostasis model assessment: insulin resistance and beta-cell function from fasting plasma glucose and insulin concentrations in man. Diabetologia. 1985;28(7):412-9.

27. Friedewald WT, Levy RI, Fredrickson DS. Estimation of the concentration of low-density lipoprotein cholesterol in plasma, without use of the preparative ultracentrifuge. Clin Chem. 1972;18(6):499 502.

28. Brasil AR, et al. C-reactive protein as an indicator of low intensity inflammation in children and adolescents with and without obesity. J Pediatr. 2007;83(5):477-80.

29. Zimmet P, Alberti KG, Kaufman F, Tajima N, Silink M, Arslanian $\mathrm{S}$, et al. The metabolic syndrome in children and adolescents - an IDF consensus report. Pediatr Diabetes. 2007;8(5):299-306.

30. Stiburkova B, et al. Metabolic syndrome, alcohol consumption and genetic factors are associated with serum uric acid concentration. PLoS One. 2014;9(5):e97646.

31. Perez-Ruiz F, Becker MA. Inflammation: a possible mechanism for a causative role of hyperuricemia/gout in cardiovascular disease. Curr Med Res Opin. 2015;31(Suppl 2):9-14.

32. Silva HA, Carraro JC, Bressan J, Hermsdorff HH. Relation between uric acid and metabolic syndrome in subjects with cardiometabolic risk. Einstein (Sao Paulo). 2015;13(2):202-8.

33. Krishnan E, Pandya BJ, Chung L, Hariri A, Dabbous O. Hyperuricemia in young adults and risk of insulin resistance, prediabetes, and diabetes: a 15-year follow-up study. Am J Epidemiol. 2012;176(2):108-16.

34. Wang J, Qin T, Chen J, Li Y, Wang L, Huang H, et al. Hyperuricemia and risk of incident hypertension: a systematic review and meta-analysis of observational studies. PLoS One. 2014;9(12):e114259.

35. Chen JH, Pan WH, Hsu CC, Yeh WT, Chuang SY, Chen PY, et al. Impact of obesity and hypertriglyceridemia on gout development with or without hyperuricemia: a prospective study. Arthritis Care Res (Hoboken). 2013;65(1):133-40.

36. Ciarla S, Struglia M, Giorgini P, Striuli R, Necozione S, Properzi G, et al. Serum uric acid levels and metabolic syndrome. Arch Physiol Biochem. 2014;120(3):119-22.

37. Ford ES, Li C, Cook S, Choi HK. Serum concentrations of uric acid and the metabolic syndrome among US children and adolescents. Circulation. 2007;115(19):2526-32.

38. Civantos Modino S, Guijarro de Armas MG, Monereo Mejías S, Montaño Martínez JM, Iglesias Bolaños P, Merino Viveros M, et al. Hyperuricemia and metabolic syndrome in children with overweight and obesity. Endocrinol Nutr. 2012;59(9):533-8. 
39. Ziemer DC, Kolm P, Weintraub WS, Vaccarino V, Rhee MK, Twombly JG, et al. Glucose-independent, black-white differences in hemoglobin A1c levels: a cross-sectional analysis of 2 studies. Ann Intern Med. 2010;152(12):770-7.

40. Chapp-Jumbo E, Edeoga C, Wan J, Dagogo-Jack S, Pathobiology of Prediabetes in a Biracial Cohort (POP-ABC) Research Group. Ethnic disparity in hemoglobin A1c levels among normoglycemic offspring of parents with type 2 diabetes mellitus. Endocr Pract. 2012;18(3):356-62.

41. Shroff R, Syrop CH, Davis W, van Voorhis B, Dokras A. Risk of metabolic complications in the new PCOS phenotypes based on the Rotterdam criteria. Fertil Steril. 2007;88(5):1389-95.

42. Donadio C, et al. Single- and multi-frequency bioelectrical impedance analyses to analyse body composition in maintenance haemodialysis patients: comparison with dual-energy x-ray absorptiometry. Physiol Meas. 2008;29(6):S517-24.
43. Haroun D, Croker H, Viner RM, Williams JE, Darch TS, Fewtrell MS, et al. Validation of BIA in obese children and adolescents and re-evaluation in a longitudinal study. Obesity (Silver Spring). 2009;17(12):2245-50.

44. Ramirez-Velez, R., et al., Validation of multi-frequency bioelectrical impedance analysis versus dual-energy X-ray absorptiometry to measure body fat percentage in overweight/obese Colombian adults. Am J Hum Biol, 2018. 30(1).

45. Nigam P, Misra A, Colles SL. Comparison of DEXA-derived body fat measurement to two race-specific bioelectrical impedance equations in healthy Indians. Diabetes Metab Syndr. 2013;7(2):72-7.

Publisher's note Springer Nature remains neutral with regard to jurisdictional claims in published maps and institutional affiliations. 